\title{
Coded Acoustic Microscopy to Study Wood Mechanics and Development
}

\author{
Hyvönen, Jere
}

IEEE

2019

Hyvönen , J , Alonso Serra , J , Meriläinen , A , Help-Rinta-Rahko , H , Nieminen , K , Salmi , A , Svedström , K , Helariutta , Y \& Haeggström , E 2019, Coded Acoustic Microscopy to Study Wood Mechanics and Development . in 2019 IEEE International Ultrasonics Symposium (IUS) . IEEE , pp. 1989-1991, IEEE International Ultrasonics Symposium , Glasgow , United Kingdom , 06/10/2019 . https://doi.org/10.1109/ULTSYM.2019.8926285

http://hdl.handle.net/10138/326126

https://doi.org/10.1109/ULTSYM.2019.8926285

acceptedVersion

Downloaded from Helda, University of Helsinki institutional repository.

This is an electronic reprint of the original article.

This reprint may differ from the original in pagination and typographic detail.

Please cite the original version. 


\section{Coded Acoustic Microscopy to Study Wood Mechanics and Development}

\author{
Jere Hyvönen \\ Electronics Research Laboratory \\ Department of Physics \\ University of Helsinki \\ Helsinki, Finalnd \\ jere.hyvonen@helsinki.fi
}

\author{
Juan Alonso Serra \\ Organismal and Evolutionary \\ Biology Research Program, \\ Faculty of Biological and \\ Environmental Sciences \\ University of Helsinki \\ Helsinki, Finland
}

Kaisa Nieminen

Production Systems, Natural

Resources Institute Finland

(Luke)

Helsinki, Finland

Restrom

Electronics Research Laboratory

Department of Physics

University of Helsinki

Helsinki, Finland
Ari Salmi

Electronics Research Laboratory

Department of Physics

Helsinki, Finland

ari.salmi@helsinki.fi
University of Helsinki

\author{
Antti Meriläinen \\ Electronics Research Laboratory \\ Department of Physics \\ University of Helsinki \\ Helsinki, Finalnd \\ antti.merilainen@helsinki.fi
}

\author{
Hanna Help-Rinta-Rahko \\ X-Ray Laboratory \\ Department of Physics \\ University of Helsinki \\ Helsinki, Finland
}

\author{
Kirsi Svedström \\ X-Ray Laboratory \\ Department of Physics \\ University of Helsinki \\ Helsinki, Finland
}

\author{
Yrjö Helariutta \\ Sainsbury Laboratory \\ University of Cambridge \\ Cambridge, United Kingdom
}

\begin{abstract}
We have developed a coded excitation scanning acoustic microscope (CESAM) that operates in range of 0.1 to 1 GHz. We used a focusing transducer with $375 \mathrm{MHz}$ central frequency to image two different tree species (birch and hybrid aspen) at different stem height to study their micromechanical difference. The method was able to capture the fresh wood anatomy with cellular resolution. A full stem section scan revealed the heterogeneity of micromechanical properties throughout tissues, and highlighted the higher stiffness of the phloem fibers compared to other vascular cells. This demonstrates the applicability of the method for plant developmental biology.
\end{abstract}

Keywords - Acoustic microscopy, Wood developmental biology, Acoustic impedance, Imaging

\section{INTRODUCTION}

The Scanning acoustic microscope (CESAM) is an instrument that employs high frequency focused ultrasound to map micromechanical properties of a sample with a few micrometer resolution. The contrast mechanism of this microscope differs from that of other instruments, e.g. optical microscopy with contrast of optical properties or atomic force microscopy (AFM) with contrast of surface elasticity. In acoustic microscopy the contrast is evident from the scattering (reflection) of acoustic waves at an acoustic impedance interface i.e. the surface of the sample. Local surface properties (stiffness tensor, density, and roughness at the scale of the wave length) define the magnitude, time-of-flight (ToF), and phase of the reflected signal. In comparison to AFM, CESAM can quickly map large areas with nearly the same small resolution. This makes CESAM a unique imaging modality to image mesoscale samples such as developing wood, which requires high resolution combined with large imaging area with a contrast mechanism based on mechanical properties. Wood is a complex composite with unique biomechanical properties. Beyond its excellent performance as raw material, little is known about how the mechanical properties of wood are acquired during the development of trees. Wood development takes place during the radial growth of a tree trunk. In this process a tissue constituted of stem cells called vascular cambium divides parallel to the stem axis and produces phloem tissues outwards, that later conform the bark, and xylem tissues inwards, commonly referred to as wood. Xylem tissues are essential to provide physical support to the stem of trees. Xylem cells are at the same time responsible for transporting water and nutrients from the roots to the rest of the plant body. Phloem tissues provide mechanical support to the bark and transport photoassimilates. During the development of these vascular tissues, plant cells produce rigid secondary cell walls, a cellular shielding not present in animals. Thus, undifferentiated tissues such as cambium or highly specialized 
tissues such as xylem or phloem are easy to differentiate anatomically, and their biomechanical properties are expected to change on the radial direction during secondary growth. We employ CESAM to study this phenomenon. In particular we dissected the stems of birch (Betula pendula) and hybrid aspen (Populus tremula $x$ tremuloides) trees, and compare their relative mechanical properties across tissues, developmental stages, and between species. The wood samples remain very close to their natural state due to minimal sample preparations.

\section{MATERIALS AND METHODS}

\section{A. Coded Excitation Scanning Acoustic Microscope (CESAM)}

Our custom-built CESAM operates in a frequency range of 0.1 to $1 \mathrm{GHz}$. We used a $375 \mathrm{MHz}$ focusing transducer $(-6 \mathrm{~dB}$ bandwidth: from 303 to $443 \mathrm{MHz}$, Kibero) to excite a 300 $\mathrm{MHz}$ to $500 \mathrm{MHz}$ linear chirp with an gaussian amplitude envelope. A detailed description of the device is found in [1].

First, we aligned the sample and the transducer with the scanning axis of the translation stage. Utilizing time-of-flight values we selected samples that could be imaged with a single C-scan. A $375 \mathrm{MHz}$ center band transducer was chosen as a compromise between resolution and working distance and was used to image the samples with single C-scans without too much defocusing. Acoustic impedance maps were calculated using acoustic impedance calibration described in [2]

\section{B. Sample Preparation}

Samples were obtained from 4 months old greenhouse grown birch (Betula pendula) and hybrid aspen (Populus tremula $x$ tremuloides) trees. Stem sections were collected from the second internode below the shoot apex (internode 2), the middle stem (half-height), and at the stem base. All stem sections were collected on the imaging day. The samples were attached to a glass using superglue (cyanoacrylate, Gorilla glue) and then immersed in ion-exchanged water at room temperature. All samples were treated with vacuum for $\sim 10$ minutes before measurements to remove air from the sample and to avoid bubble formation that would have distorted the images.

\section{RESULTS}

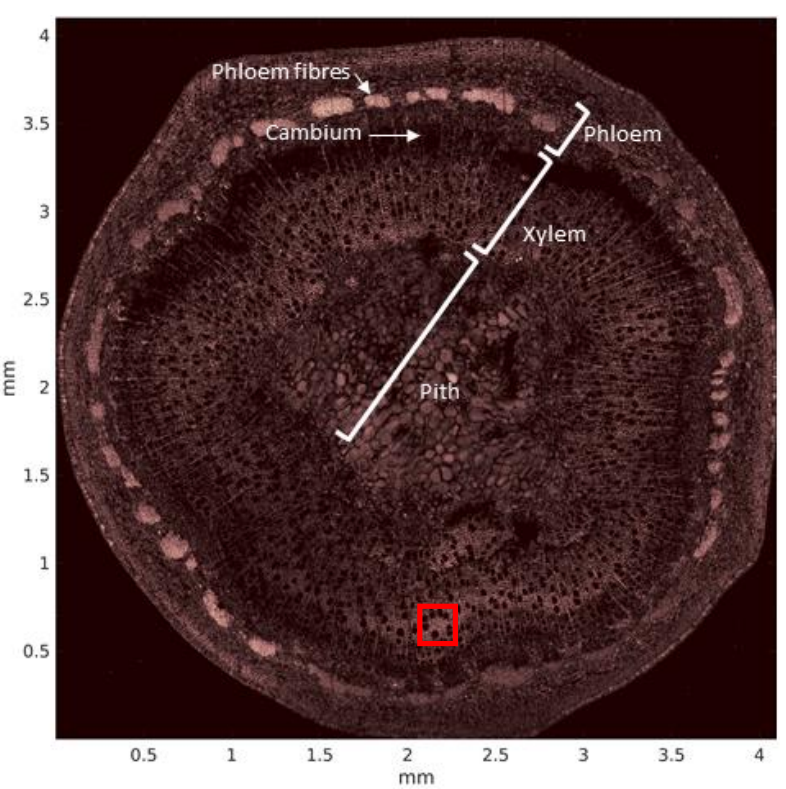

Fig.1. $4 \times 4 \mathrm{~mm}^{2}$ amplitude C-scan with $1 \mu \mathrm{m}$ steps of the crosssection of an aspen stem cut from the middle height of the tree. Imaging time $45 \mathrm{~min}$. The red square marks the area of Fig.2.

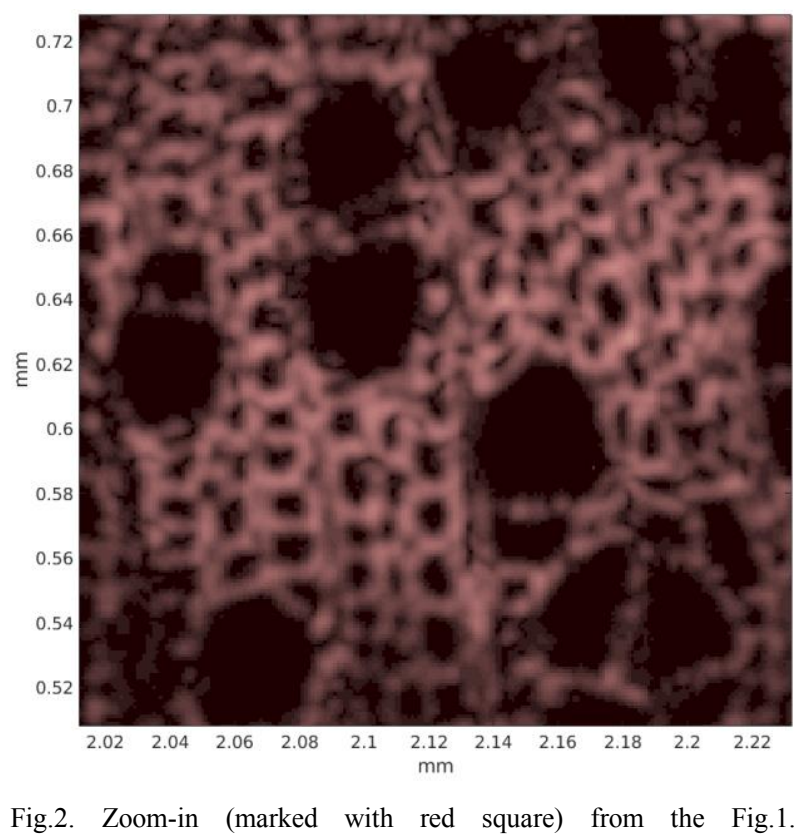




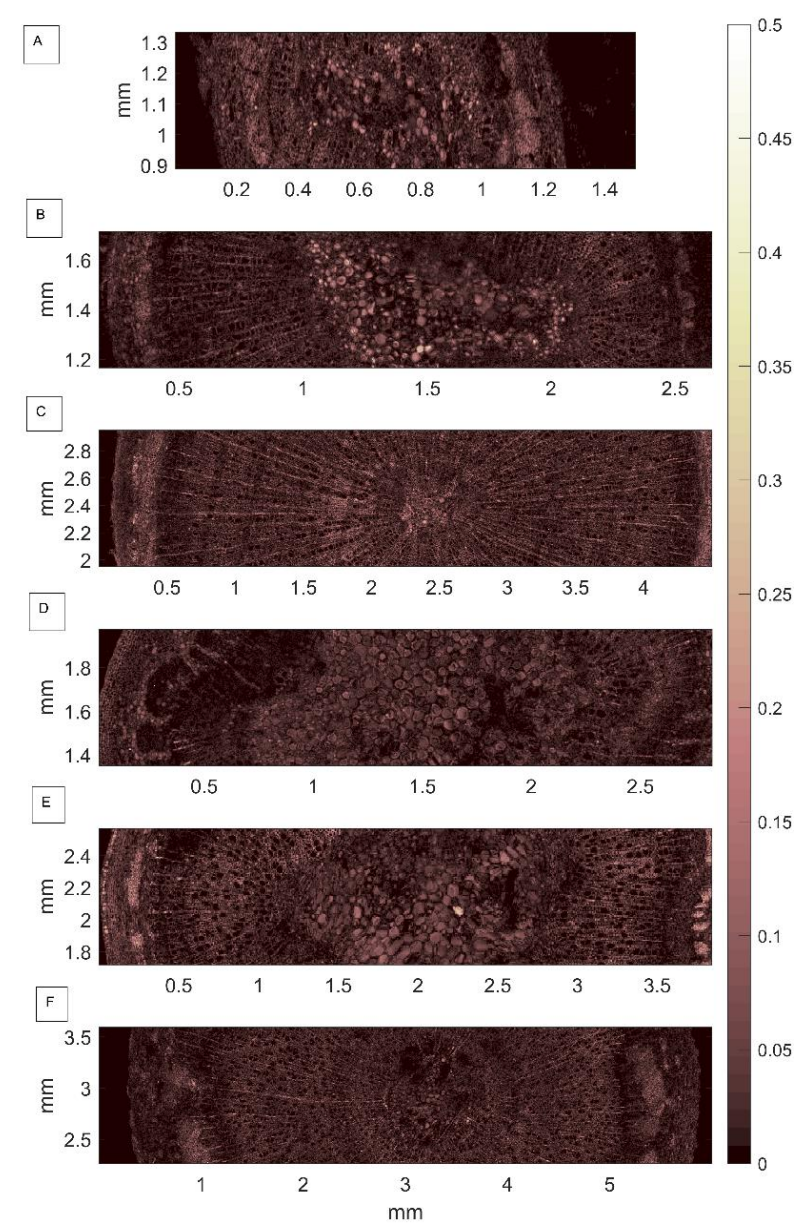

Fig.3. Amplitude C-Scans from different heights of both species. A) Birch $2^{\text {nd }}$ internode. B) Birch middle height C) Base of the stem of a birch. D) $2^{\text {nd }}$ internode of an aspen. E) Aspen middle height. F). Base of the stem of an aspen

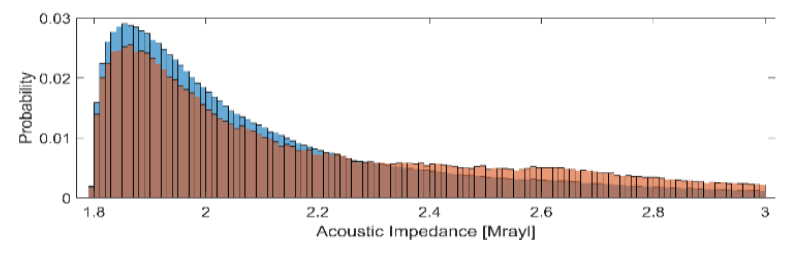

Fig. 4. Acoustic impedance histograms of xylem tissue from $2^{\text {nd }}$ internodes of birch (red) and aspen (blue).

\section{DISCUSSION}

The focal echo amplitude is proportional to the local acoustic impedance of the tissue. The value measured is lowered by possible defocusing and scattering. The acoustic impedance estimations in Fig. 4 have smaller values than the $\mathrm{V}(\mathrm{z})$-analysis done by Dawei $\mathrm{Wu}$ et al. [6] possibly due to sample being non-ideal reflector. Also, fresh cells have high water content which affects the acoustic interface of reflection. The echo amplitude maps revealed with cellular resolution the tissue-specific focal mechanics of both species. Hybrid aspen and silver birch are model organisms of angiosperm tree species [4]. In both species the early stages of wood development can be appreciated in the internode 2 (fig. 1, fig.
2, and fig. 3. B, E). In this section the largest area of the stem is occupied by the pith that is rich in parenchymatic cells. This cell type lacks secondary cell walls, therefore the apparent stiffness in some pith cells may be a cause of their high water content. Wood at this developmental stage consist of primary xylem that is rich in vessels, water transporting cells with secondary cell walls. On the phloem side of the cambium phloem fibers appear to have a higher amplitude indicative of higher mechanical stiffness compared to any other bark tissue. This characteristic is more evident in birch compared to aspen sections of internode 2. Interestingly, phloem fibers appear to be from this perspective, as stiff as or even stiffer than the xylem tissues themselves. The contribution of this cell type to mechanical functions in the stem of trees has recently been revisited, suggesting that they provide longitudinal postural control [3]. Along the radial direction the sections of both species showed a cylindrical ring of lower focal echo amplitude that coincides with the vascular cambium and developing xylem tissues. Towards the base of the stem were tissues are more mature, this cambial ring is more irregular in hybrid aspen and more cylindrical in silver birch. Additionally, phloem fibers at the stem base appear to be arranged in large isolated clusters of cells in hybrid aspen but forming nearly complete rings in silver birch. Combined these observations suggest that the different anatomical organization across species or developmental stages is also reflected in a variable mechanical architecture inside the stem of trees.

\section{CONCLUSIONS}

The application of CESAM on stem cross-sections revealed for the first time the relative differences in mechanical properties across vascular tissues in trees. The support function of trees is typically attributed to xylem tissues. While this is the case in adult trees were xylem occupies a larger sectional area, the mechanical maps presented here further encourage us to study the contribution of additional cell types, such as phloem fibers, to the mechanical stability of stems in early stages of tree development.

\section{REFERENCES}

[1] Hyvönen et al, "Three Megapixel Scanning Acoustic Microscope Imaging”, UFFC IUS, Glasgow 2019

[2] K. Raum, K. V. Jenderka, A. Klemenz and J. Brandt, "Multilayer analysis: quantitative scanning acoustic microscopy for tissue characterization at a microscopic scale," in IEEE Transactions on Ultrasonics, Ferroelectrics, and Frequency Control, vol. 50, no. 5, pp. 507-516, May 2003.

[3] Clair, B., Ghislain, B., Prunier, J., Lehnebach, R., Beauchêne, J. and Alméras, T. 2019. Mechanical contribution of secondary phloem to postural control in trees: the bark side of the force. The New Phytologist 221(1), pp. 209-217.

[4] Salojärvi, J., Smolander, O.-P., Nieminen, K., et al. 2017. Genome sequencing and population genomic analyses provide insights into the adaptive landscape of silver birch. Nature Genetics 49(6), pp. 904-912.

[5] Tuskan, G.A., Difazio, S., Jansson, S., et al. 2006. The genome of black cottonwood, Populus trichocarpa (Torr. \& Gray). Science 313(5793), pp. 1596-1604.

[6] Dawei Wu, R. Petherick and P. Harris, "Measurement of local wood velocites by acoustic microscopy," 2013 IEEE International Ultrasonics Symposium (IUS), Prague, 2013, pp. 1587-1589. doi: 10.1109/ULTSYM.2013.0404 